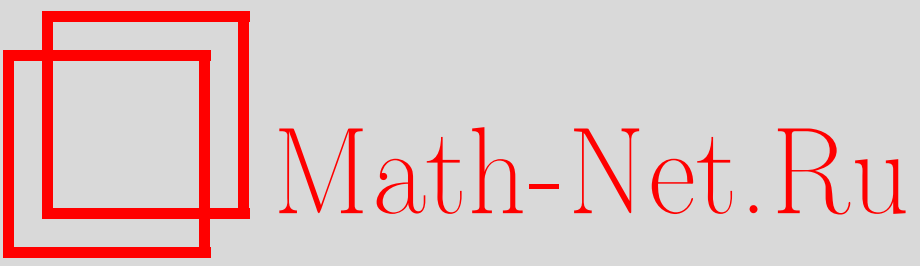

В. Р. Халилов, Поляризация вакуума $(2+1)$-мерной квантовой электродинамики с ненулевой плотностью фермионов внешним магнитным полем, ТМФ, 2000, том 125, номер 1, 132-151

DOI: https://doi.org/10.4213/tmf661

Использование Общероссийского математического портала Math-Net.Ru подразумевает, что вы прочитали и согласны с пользовательским соглашением

http: //www. mathnet.ru/rus/agreement

Параметры загрузки:

IP : 52.90 .164 .192

26 апреля 2023 г., 04:53:32 


\section{ПОЛЯРИЗАЦИЯ ВАКУУМА $(2+1)$ МЕРНОЙ КВАНТОВОЙ ЭЛЕКТРОДИНАМИКИ С НЕНУЛЕВОЙ ПЛОТНОСТЬЮ ФЕРМИОНОВ ВНЕШНИМ МАГНИТНЫМ ПОЛЕМ}

Построена функция Грина уравнения Дирака во внешнем постоянном и однородном магнитном поле в $(2+1)$-мерной квантовой электродинамике $\left(\mathrm{K} Д_{2+1}\right)$ с ненулевой плотностью фермионов. В однопетлевом приближении КЭД $2+1$ получено выражение для поляризационного оператора во внешнем постоянном и однородном магнитном поле при ненулевом химическом потенциале. Вычислен вклад индуцированного члена Черна-Саймонса в поляризационный оператор и эффективный лагранжиан при плотности фермионов, соответствующей заполнению ими $n$ релятивистских уровней Ландау во внешнем магнитном поле. Получено выражение для индуцированного члена Черна-Саймонса в магнитном поле при конечной температуре и ненулевом химическом потенциале.

\section{1. ВВЕДЕНИЕ}

В последние годы значительно возрос интерес к эффектам электродинамики в $(2+1)$ измерениях. Этот интерес стимулирован необходимостью изучения квантовых состояний электронов на границе раздела двух сред и целым рядом эффектов в физике конденсированных сред, указывающих на существование квантовых систем, которые хорошо описываются уравнением Дирака в $(2+1)$ измерениях [1]. Двумерные системы нерелятивистских заряженных фермионов, связанные с калибровочным электромагнитным полем и калибровочным полем Черна-Саймонса, также привлекают внимание в связи с их свойствами, позволяющими применять эти модели к изучению таких квантовых макроскопических явлений, как квантовый эффект Холла и высокотемпературная сверхпроводимость [2-5].

Свойства квантовых $(2+1)$-мерных фермионов вызывают и чисто теоретический интерес в связи с теорией анионов - частиц, подчиняющихся дробной статистике в $(2+1)$ измерениях [6-8]. Проблемы нестабильности непертурбативного вакуума и возникновения спонтанной намагниченности в $(2+1)$-мерной квантовой электродинамике, индуцированной присутствием “затравочного” члена Черна-Саймонса, обсуждались в ра-

\footnotetext{
* Московский государственный университет, Москва, Россия. E-mail: khalilov@thc.phys.msu.su
} 
боте [9]. Эффект рождения пар заряженных фермионов из вакуума сильным кулоновским полем протяженного источника в $(2+1)$ измерениях рассмотрен в [10]. Некоторые аналитические решения уравнений Шредингера и Дирака во внешних кулоновском и магнитном полях в $(2+1)$ измерениях, которые представимы в виде произведения весовой функции и конечного полинома, найдены в [11]. Показано, что такие аналитические решения и соответствуюший им спектр энергий существуют при некоторых определенных соотношениях между параметрами, характеризуюшими интенсивность взаимодействия заряженного фермиона с кулоновским и магнитным полями, для бесчисленного множества значений напряженности магнитного поля. Такая квантово-механическая система представляет собой пример открытых недавно “квазиточно" решаемых моделей [12]. Значительный интерес могут представить эффекты, возникаюшие в присутствии постоянного однородного магнитного поля при конечной температуре [13].

Напомним, что радиационные эффекты определяют массовый оператор и уровни энергии электрона в рассматриваемом внешнем поле. Более того, свойства электромагнитных взаимодействий заряженных частиц во внешних электромагнитных полях с учетом радиационных поправок могут быть изучены с помошью поляризационного оператора. Впервые аналитические свойства поляризационного оператора во внешних постоянных электромагнитных полях различных конфигураций в однопетлевом приближении КЭД $3+1$ были изучены в работах [14] и [15]. Интересные эффекты, обусловленные поляризацией вакуума КЭД $3+1$ внешним нестационарным электромагнитным полем, изучались в [16]. Антисимметричная часть поляризационного оператора во внешнем постоянном однородном магнитном поле при ненулевой плотности фермионов в однопетлевом приближении КЭД $2+1$ в пределе, когда 3 -импульс фотона стремится к нулю, определяет общее выражение для электрической проводимости в $\mathrm{K} \beth_{2+1}$ [17].

Значительную информацию о свойствах электромагнитных взаимодействий заряженных фермионов во внешних электромагнитных полях с учетом радиационных поправок можно получить с помошью эффективного лагранжиана $[16,18]$.

В данной работе изучается модель $(2+1)$-мерной квантовой электродинамики в аспекте физики конденсированных сред. А именно, рассматривается модель КЭД $2+1$ с ненулевым химическим потенциалом $\mu$ в присутствии внешнего постоянного и однородного магнитного поля, описываемая лагранжианом

$$
L=\bar{\psi}\left[\gamma^{\mu}\left(i \partial_{\mu}-e A_{\mu}\right)+\gamma_{0} \mu-m\right] \psi-\frac{1}{4} F_{\mu \nu} F^{\mu \nu} .
$$

Здесь $F_{\mu \nu}=\partial_{\mu} A_{\nu}-\partial_{\nu} A_{\mu}$ - тензор электромагнитного поля, причем $A_{\mu}=A_{\mu}^{(\mathrm{e})}+A_{\mu}^{(\mathrm{q})}$, где $A_{\mu}^{(\mathrm{e})}$ - внешнее, $A_{\mu}^{(\mathrm{q})}-$ квантованное электромагнитные поля, а параметр $m$ имеет смысл массы фермиона. Известно (см., например, [4]), что в $(2+1)$ измерениях алгебра матриц Дирака может быть представлена с помошью матриц Паули. Мы выберем представление $\gamma^{0}=\sigma_{3}, \gamma^{k}=i \sigma_{k}$. Метрический тензор пространства Минковского имеет вид $g^{\mu \nu}=g_{\mu \nu}=\operatorname{diag}(1,-1,-1)$. Массовый член в модели (1) неинвариантен относительно операций пространственной четности и обрашения времени, поэтому член Черна-Саймонса вида [19]

$$
L_{\mathrm{CS}}=\frac{e^{2} \operatorname{sign} m}{16 \pi} e^{\mu \nu \rho} F_{\mu \nu} A_{\rho}
$$


в эффективном лагранжиане Гайзенберга-Эйлера для рассматриваемой модели должен генерироваться в вакууме внешним полем "динамически", несмотря на то что такой член отсутствует в “затравочном" лагранжиане (1). В формуле (2) $e^{\mu \nu \rho}$ есть полностью антисимметричный единичный псевдотензор 3-го ранга, у которого отличны от нуля лишь компоненты с тремя различными индексами. При перестановке любых двух индексов компоненты псевдотензора $e^{\mu \nu \rho}$ меняют знак, и мы полагаем, что $e^{012}=1$.

В этой работе будет найдена функция Грина уравнения Дирака в присутствии внешнего постоянного магнитного поля в КЭД $2+1$ с ненулевой плотностью фермионов. Используя построенную функцию Грина, мы получим выражение для поляризационного оператора во внешнем постоянном магнитном поле при ненулевом химическом потенциале в однопетлевом приближении КЭД $2+1$. Мы вычислим вклад индуцированного члена Черна-Саймонса в поляризационный оператор и эффективный лагранжиан при плотности фермионов, соответствующей заполнению ими $n$ релятивистских уровней Ландау во внешшем магнитном поле, а также получим выражение для индуцированного члена Черна-Саймонса при конечной температуре и при ненулевом химическом потенциале. Наконец, мы обсудим некоторые эффекты, обусловленные присутствием члена Черна-Саймонса в эффективном лагранжиане.

В статье используется система единиц, в которой $c=\hbar=1$.

\section{2. РЕШЕНИЯ УРАВНЕНИЯ ДИРАКА В МАГНИТНОМ ПОЛЕ}

Движение заряженного фермиона массы $m$ и заряда $e$ в $(2+1)$ измерениях во внешнем электромагнитном поле будем описывать уравнением Дирака, записанным в ковариантном виде

$$
\left(\gamma^{\mu} P_{\mu}-m\right) \Psi=0
$$

Здесь $P_{\mu}=p_{\mu}-e A_{\mu} \equiv\left(P_{0}, P_{1}, P_{2}\right)$ - оператор обобщенного импульса фермиона, $p_{\mu}=$ $\left(i \partial_{t},-i \partial_{x},-i \partial_{y}\right) \equiv\left(p_{0}, p_{1}, p_{2}\right)$. Разумеется, вектор-потенциал $A_{\mu}$ описывает только внешнее магнитное поле. Вектор-потенциал магнитного поля выбран в калибровке Ландау $A_{0}=A_{1}=0, A_{2}=B x$, а внешнее магнитное поле определено как $B=\partial_{1} A_{2}-\partial_{2} A_{1}$. Решение уравнения Дирака (3) можно найти методом собственных функций во внешнем электромагнитном поле, который был развит Ритусом в работе [18] для случая $\mathrm{K} Э Д_{3+1}$.

Введем матричную функцию $E_{p}$, удовлетворяюшую уравнению с квадрированным оператором Дирака [18]:

$$
(\gamma P)^{2} E_{p} \equiv\left(P_{0}^{2}-P_{1}^{2}-P_{2}^{2}-e \sigma_{3} B\right) E_{p}=p^{2} E_{p},
$$

где собственное значение $p^{2}$ - любое вешественное число. Функция $E_{p}$ является также собственной функцией следуюших операторов:

$$
i \partial_{t} E_{p}=p_{0} E_{p}, \quad-i \partial_{y} E_{p}=p_{2} E_{p}, \quad\left(P_{1}^{2}+P_{2}^{2}-e \sigma_{3} B\right) E_{p}=2|e B| k E_{p} .
$$

Эти операторы коммутируют с $(\gamma P)$, а их собственные значения $p_{0}, p_{2}$ и $2|e B| k$ нумеруют решения уравнения Дирака (3). Операторы (4) и (5) коммутируют с $\sigma_{3}$, и поэтому 
их собственные функции также различаются собственными значениями $\sigma= \pm 1$ оператора $\sigma_{3}$.

Собственные функции $E_{p \sigma}(z)$ имеют вид

$$
E_{p \sigma}(z)=\frac{|e B|^{\frac{1}{4}}}{(\pi)^{\frac{1}{4}}(n !)^{\frac{1}{2}}} e^{-i p_{0} t+i p_{2} y} D_{n}(\eta) w_{\sigma},
$$

где $w_{\sigma}$ - собственные векторы матрицы $\sigma_{3}, D_{n}(\eta)$ - функции параболического цилиндра с индексом $n=k+(\sigma / 2) \operatorname{sign}(e B)-1 / 2, n=0,1, \ldots$, и аргументом $\eta=\sqrt{2|e B|}(x-$ $\left.p_{2} / e B\right)$. Два спинора $w_{1}$ и $w_{-1}$ образуют первый и второй столбцы единичной матрицы в пространстве $\gamma$-матриц, т.е. $w_{\sigma}(\alpha)=I_{\alpha \beta}$, если собственным значениям $\sigma$ сопоставить номер столбца.

Матричные функции $E_{p}(z)$ ортогональны:

$$
\int \bar{E}_{p^{\prime}}(z) E_{p}(z) d^{3} z=\delta\left(p_{0}^{\prime}-p_{0}\right) \delta\left(p_{2}^{\prime}-p_{2}\right) \delta_{k^{\prime} k}
$$

и удовлетворяют условию полноты

$$
\sum_{k=0}^{\infty} \int d p_{0} d p_{2} E_{p}(z) \bar{E}_{p}\left(z^{\prime}\right)=\delta\left(z-z^{\prime}\right) .
$$

В формулах (7) и (8) $\bar{E}_{p}=\gamma^{0} E_{p}^{\dagger} \gamma^{0}$, а $E_{p}^{\dagger}$ - эрмитово-сопряженная матричная функция. Так как [18]

$$
(\gamma P) E_{p}=E_{p}(\gamma \bar{p})
$$

то “3-вектор" $\bar{p}_{\mu}$ имеет компоненты

$$
\bar{p}_{\mu}=\left(p_{0}, 0, \operatorname{sign}(e B) \sqrt{2|e B| k}\right),
$$

а $p^{2}=\bar{p}^{2}$. Вектор $\bar{p}$ зависит только от “динамических" квантовых чисел $p_{0}$ и $k$.

Решение уравнения Дирака (3) можно получить, подействовав оператором $(\gamma P+m)$ на $E_{p}$-матрицу, взятую при $p^{2}=m^{2}$,

$$
\Psi_{p}(z)=(\gamma P+m) E_{p}=E_{p}(\gamma \bar{p}+m) .
$$

Решение имеет вид

$$
\Psi_{p}(z)=E_{p}(z) u_{\bar{p}}
$$

где $u_{\bar{p}}-$ спинор, удовлетворяющий свободному уравнению Дирака

$$
(\gamma \bar{p}-m) u_{\bar{p}}=0
$$

а матрицу $E_{p}$ следует взять при $p^{2}=m^{2}$.

Спектр энергий и нормированные решения уравнения (13) имеют вид

$$
\begin{gathered}
\bar{p}_{0} \equiv E_{k}=\sqrt{m^{2}+2|e B| k} \\
\left(\begin{array}{c}
u_{\bar{p}}^{1} \\
u_{\bar{p}}^{2}
\end{array}\right) \equiv\left(\begin{array}{c}
u_{+}^{1} \\
u_{+}^{2}
\end{array}\right)=\frac{1}{\sqrt{2 E_{k}}}\left(\begin{array}{c}
\sqrt{E_{k}+m} \\
-\operatorname{sign}(e B) \sqrt{E_{k}-m}
\end{array}\right) .
\end{gathered}
$$


Решения (12), (15), взятые с матрицами (6), представляют собой положительно-частотные решения уравнения Дирака.

Уравнение для спинора $u_{-\bar{p}}$, описываюшее решения уравнения Дирака с отрицательными энергиями, можно получить из уравнения (13), заменив в последнем оператор $\bar{p}$ на $-\bar{p}$ :

$$
(\gamma \bar{p}+m) u_{-\bar{p}}=0 .
$$

Нормированные решения уравнения (16) имеют вид

$$
\left(\begin{array}{c}
u_{-\bar{p}}^{1} \\
u_{-\bar{p}}^{2}
\end{array}\right) \equiv\left(\begin{array}{c}
u_{-}^{1} \\
u_{-}^{2}
\end{array}\right)=\left(\begin{array}{c}
u_{+}^{2} \\
u_{+}^{1}
\end{array}\right) .
$$

Решения (12), (17) с комплексно-сопряженными матрицами (6) представляют решения уравнения Дирака с отрицательными частотами. Заметим, что отрицательно-частотные решения уравнения Дирака, описываюшие античастицу, можно получить из решений (12) и (13) операцией зарядового сопряжения.

Найденные решения являются собственными функциями оператора $(\gamma P)^{2}-P_{0}^{2}$ :

$$
\left[(\gamma P)^{2}-P_{0}^{2}\right] \Psi_{p \sigma}=(|e B|(2 k+1)+e B \sigma) \Psi_{p \sigma}, \quad k=0,1, \ldots,
$$

и оператора $f=\sigma_{3} / 2$ :

$$
f \Psi_{p \sigma}=\left(\frac{\sigma}{2}\right) \Psi_{p \sigma}, \quad \sigma= \pm 1 .
$$

Все уровни энергии, за исключением уровня с $k=0, \sigma=-1$ (для $\operatorname{sign}(e B)>0$ ) и уровня с $k=0, \sigma=+1$ (для $\operatorname{sign}(e B)<0$ ), двукратно вырожденны: совпадают уровни с $k$, $\sigma=+1$ и $k+1, \sigma=-1$ (для $\operatorname{sign}(e B)>0$ ) и уровни с $k, \sigma=-1$ и $k+1, \sigma=+1$ (для $\operatorname{sign}(e B)<0)$. Оператор $f=\sigma_{3} / 2$ имеет смысл оператора фермионного числа в суперсимметричной модели квантовой механики [20]. Собственные функции гамильтониана уравнения Дирака не являются собственными функциями оператора $\sigma_{3}$.

Состояния электрона в постоянном магнитном поле характеризуем положительно-частотными решениями уравнения Дирака

$$
\Psi^{+}(t, \mathbf{x})=\exp \left(-i E_{n}^{+} t\right) \psi_{n}^{+}(\mathbf{x}) \equiv \exp \left(-i E_{n}^{+} t+i p_{2} y\right)\left(\begin{array}{c}
\psi_{+}^{1}(x) \\
\psi_{+}^{2}(x)
\end{array}\right)
$$

соответствующими собственному значению энергии (уровень Ландау) $E_{n}^{+}=E_{n} \equiv$ $\sqrt{m^{2}+2 n|e H|}$. Две компоненты зависяшего от $x$ спинора $\psi(x)$, найденные выше, приведем в явном виде:

$$
\left(\begin{array}{c}
\psi_{+}^{1}(x) \\
\psi_{+}^{2}(x)
\end{array}\right)=\left(\begin{array}{c}
u_{+}^{1}(n) U_{n}(\eta) \\
u_{+}^{2}(n) U_{n-1}(\eta)
\end{array}\right)
$$

Здесь $u_{+}^{1}(n), u_{+}^{2}(n)$ определяются формулой $(15)$, в которой индекс $k$ следует заменить индексом $n$. Нормированные функции

$$
U_{n}(\eta)=\frac{|e B|^{\frac{1}{4}}}{\left(2^{n} n ! \pi^{\frac{1}{2}}\right)^{\frac{1}{2}}} \exp \left(-\frac{\eta^{2}}{4}\right) H_{n}\left(\frac{\eta}{\sqrt{2}}\right)
$$


выражены через полиномы Эрмита $H_{n}(\eta / \sqrt{2})$ с использованием формулы [21]

$$
D_{n}(z)=2^{-\frac{n}{2}} e^{-\frac{z^{2}}{4}} H_{n}\left(\frac{z}{\sqrt{2}}\right) .
$$

Нормированные решения уравнения Дирака, относяшиеся к отрицательным энергиям $\left(E_{n}^{-}=-E_{n}\right)$, можно получить из положительно-частотных решений операцией зарядового сопряжения:

$$
\Psi^{-}(t, \mathbf{x})=\exp \left(i E_{n}^{-} t\right) \psi_{n}^{-}(\mathbf{x}) \equiv \exp \left(i E_{n}^{-} t-i p_{2} y\right)\left(\begin{array}{c}
\psi_{-}^{1}(x) \\
\psi_{-}^{2}(x)
\end{array}\right)
$$

где

$$
\left(\begin{array}{c}
\psi_{-}^{1}(x) \\
\psi_{-}^{2}(x)
\end{array}\right)=\left(\begin{array}{c}
u_{-}^{1}(n) U_{n-1}(\eta) \\
u_{-}^{2}(n) U_{n}(\eta)
\end{array}\right),
$$

а $u_{-}^{1}(n), u_{-}^{2}(n)$ определяются формулами (17), (15), где индекс $k$ нужно заменить индексом $n$

Выражения для операторов поля фермионов в схеме вторичного квантования можно записать в виде следуюших разложений:

$$
\begin{aligned}
& \psi=\sum_{n}\left[a_{n} \psi_{n}^{+} \exp \left(-i E_{n}^{+} t\right)+b_{n}^{+} \psi_{n}^{-} \exp \left(i E_{n}^{-} t\right)\right], \\
& \bar{\psi}=\sum_{n}\left[a_{n}^{+} \bar{\psi}_{n}^{+} \exp \left(i E_{n}^{+} t\right)+b_{n} \bar{\psi}_{n}^{-} \exp \left(-i E_{n}^{-} t\right)\right] .
\end{aligned}
$$

Здесь $\bar{\psi}=\psi^{\dagger} \gamma^{0}$, а $\psi^{\dagger}$ - эрмитово-сопряженный спинор. Операторы уничтожения фермионов $a_{n}$, как и операторы уничтожения антифермионов $b_{n}$, стоят в виде коэффициентов при найденных нормированных решениях одночастичного уравнения Дирака. Операторы поля фермионов удовлетворяют стандартным антикоммутационным соотношениям.

\section{3. ФУНКЦИЯ ГРИНА ФЕРМИОНА В МАГНИТНОМ ПОЛЕ}

Функция Грина уравнения Дирака в постоянном магнитном поле имеет особенно простой вид в $E_{p}$-представлении:

$$
S_{\mathrm{F}}(p)=\frac{1}{\gamma p-m+i \delta_{e}} \equiv \frac{\gamma p+m}{\bar{p}^{2}-m^{2}+i \delta_{e}} .
$$

Здесь согласно правилу Фейнмана введен “причинный” фактор $i \delta_{e}\left(c \delta_{e}>0\right)$, смешаюший полюсы $p_{0}= \pm E_{k} \equiv \pm \sqrt{m^{2}+2|e B| k}$ с вешественной оси $p_{0}$.

Функция Грина уравнения Дирака в так называемом собственно временном представлении [22, 23] была найдена в [24]:

$$
\begin{aligned}
S^{\mathrm{c}}\left(z, z^{\prime}\right)= & -\frac{i^{\frac{1}{2}} e B}{8 \pi^{\frac{3}{2}}} \int_{0}^{\infty} \frac{d s}{s^{\frac{1}{2}} \sin \alpha} \exp \left[-i\left(m^{2}-i \delta_{e}\right) s-\frac{i\left(t-t^{\prime}\right)^{2}}{4 s}+\right. \\
& \left.+\frac{i \alpha\left(\left(x-x^{\prime}\right)^{2}+\left(y-y^{\prime}\right)^{2}\right)}{4 s \operatorname{tg} \alpha}+\frac{i e B\left(x+x^{\prime}\right)\left(y-y^{\prime}\right)}{2}\right] \times \\
& \times\left[\left(\frac{\gamma^{0}\left(t-t^{\prime}\right)}{2 s}+m\right) e^{i \sigma_{3} \alpha}-\frac{\alpha\left[\gamma^{1}\left(x-x^{\prime}\right)+\gamma^{2}\left(y-y^{\prime}\right)\right]}{2 s \sin \alpha}\right],
\end{aligned}
$$


где $\alpha=e B s$.

Для построения функции Грина уравнения Дирака при ненулевой плотности фермионов необходимо предварительно найти функцию Грина в импульсном представлении. Для этого, выполняя интегрирование по времени и координатам в (26) с использованием интегралов

$$
\begin{aligned}
\int_{-\infty}^{\infty} e^{-\frac{i t^{2}}{4 s}-i p_{0} t} d t & =\sqrt{-2 i \pi s} e^{i s p_{0}^{2}} \\
\int_{-\infty}^{\infty} e^{i \alpha \operatorname{ctg} \alpha\left(\frac{z^{2}}{4 s}\right)+i p_{1} z} d z & =\sqrt{\frac{2 i \pi s}{\alpha \operatorname{ctg} \alpha}} e^{-\frac{i s p_{1}^{2} \operatorname{tg} \alpha}{\alpha}},
\end{aligned}
$$

получим

$$
\begin{aligned}
S^{\mathrm{c}}(p)= & -\frac{i}{|e B|} \int_{0}^{\infty} \frac{d x}{\cos x} \exp \left[\frac{i x}{|e B|}\left(p_{0}^{2}-m^{2}+i \delta_{e}-\mathbf{p}^{2} \frac{\operatorname{tg} x}{x}\right)\right] \times \\
& \times\left[\left(\gamma^{0} p^{0}+m\right) e^{i \sigma_{3} x}-\frac{\left(\gamma^{1} p^{1}+\gamma^{2} p^{2}\right)}{\cos x}\right],
\end{aligned}
$$

где мы положили $x \equiv|e B| s$. В выражении (29) сдвинем переменную $p_{0}$ следуюшим образом $p_{0} \rightarrow p_{0}+\mu+i \operatorname{sign}\left(p_{0}\right) \epsilon$, где $\mu-$ химический потенциал [25]. Случаю заполнения релятивистских уровней Ландау в магнитном поле реальными частицами соответствуют значения $\mu>m$. Если $\mu<m$, то реальных частиц нет. Далее без ограничения общности рассмотрения мы будем подразумевать, что $\mu>0$.

Окончательное выражение для функции Грина уравнения Дирака при ненулевой плотности фермионов принимает вид

$$
\begin{aligned}
S^{\mathrm{c}}\left(p^{0}, \mathbf{p}, \mu\right)= & -\frac{i}{|e B|} \int_{0}^{\infty} \frac{d x}{\cos x} \exp \left[\frac { i x } { | e B | } \left(\left(p_{0}+\mu+i \operatorname{sign}\left(p_{0}\right) \epsilon\right)^{2}-\right.\right. \\
& \left.\left.-m^{2}+i \delta_{e}-\mathbf{p}^{2} \frac{\operatorname{tg} x}{x}\right)\right] \times \\
& \times\left[\left(\gamma^{0}\left(p^{0}+\mu\right)+m\right) e^{i \sigma_{3} x}-\frac{\left(\gamma^{1} p^{1}+\gamma^{2} p^{2}\right)}{\cos x}\right]
\end{aligned}
$$

Заметим, что особенности подынтегрального выражения в (30) обходятся снизу. В физических задачах при различных интегрированиях с участием функции Грина наличие бесконечно малой мнимой части $i \operatorname{sign}\left(p_{0}\right) \epsilon$ сушественно вблизи полюсов, когда $p_{0}=$ $\pm E_{n}-\mu[25]$, поэтому sign $p_{0}$ в функции Грина можно заменить на $\operatorname{sign}\left( \pm E_{n}-\mu\right)$. В таком виде функция Грина оказьвается единой аналитической функцией во всей плоскости комплексной переменной $p_{0}[25]$.

Свойства функции Грина (30) с химическим потенциалом $\mu$ в импульсном представлении состоят в том, что, во-первых, ее полюсы могут лежать только в точках $p_{0}= \pm E_{n}-$ $\mu$, где $E_{n}$ - дискретные уровни Ландау, и, во-вторых, что она имеет мнимую часть, обусловленную присутствием реальных фермионов. Как функция переменной $p_{0}$ она является предельным значением некоторой аналитической функции $S\left(p_{0}\right)$. Действительно, поворачивая контур интегрирования по $x$ в интеграле (30) в нижнюю полуплоскость, 
можно распространить $S^{\mathrm{c}}\left(p^{0}, \mathbf{p}, \mu\right) \equiv S\left(p^{0}\right)$ на всю плоскость комплексной переменной $p_{0}$ с разрезами $\left[p_{+}, \infty\right),\left[-p_{-},-\infty\right)$ на действительной оси. Если обозначить через $S(x)$ подынтегральное выражение в $(30)$, то значения $S\left(p^{0}\right)$ на физическом и нефизическом берегах разрезов определяются как $\int_{0}^{\infty} S(x) d x$ и $\int_{0}^{-\infty} S(x) d x$, соответственно. Тогда скачки на разрезах $\Delta S$ равны

$$
\int_{-\infty}^{\infty} S(x) d x
$$

Представим мнимую часть функции Грина (скачки $\Delta S$ ) в виде суммы вкладов состояний реальных фермионов, которые заполняют уровни Ландау при ненулевой плотности фермионов. Подобный прием использовался для вычисления скачков поляризационного оператора в $\mathrm{K}$ Д $_{3+1}$ в постоянном однородном магнитном поле в [26] и в случае суперпозиции постоянного магнитного поля и поля плоской электромагнитной волны в [27].

Введем векторы

$$
\begin{aligned}
P^{0} & =\bar{p}_{0} \cos x+i m \sin x \\
M=m \cos x+i \bar{p}_{0} \sin x & \equiv \frac{1}{2}\left(\bar{p}_{0}+m\right) e^{i x}+\frac{1}{2}\left(\bar{p}_{0}+m\right) e^{i x}-\frac{1}{2}\left(\bar{p}_{0}-m\right) e^{-i x},
\end{aligned}
$$

где мы обозначили $\bar{p}_{0} \equiv p_{0}+\mu$.

С учетом этих обозначений функция Грина (30) примет вид

$$
\begin{aligned}
S^{\mathrm{c}}\left(\bar{p}_{0}, \mathbf{p}, m\right)= & -\frac{i}{|e B|} \int_{0}^{\infty} \frac{d x}{\cos x}[(\gamma P)+M] \times \\
& \times \exp \left[\frac{i x}{|e B|}\left(\left(\bar{p}_{0}+i \operatorname{sign}\left(\bar{p}_{0}\right) \epsilon\right)^{2}-m^{2}+i \delta_{e}-\mathbf{p}^{2} \frac{\operatorname{tg} x}{x}\right)\right],
\end{aligned}
$$

здесь $\mathbf{P}=-\mathbf{p} / \cos x$.

Процедуру нахождения искомого представления покажем на примере интеграла

$$
J=-\frac{i}{|e B|} \int_{-\infty}^{\infty} \frac{d x}{\cos x} \exp \left[\frac{i x}{|e B|}\left(\left(p_{0}+\mu\right)^{2}-m^{2}-\mathbf{p}^{2} \frac{\operatorname{tg} x}{x}\right)\right] .
$$

Для этого разложим экспоненту в (35) в ряд по степеням

$$
-i \frac{\mathbf{p}^{2}}{|e B|} \operatorname{tg} x \equiv-i z \operatorname{tg} x
$$

и представим тригонометрические функции в числителе в виде экспонент. Тогда получим

$$
J=-\frac{i}{|e B|} \sum_{l=0}^{\infty} \int_{-\infty}^{\infty} \frac{d x}{\cos ^{l+1} x} \sum_{k=0}^{l} \frac{(-z)^{l}(-1)^{k}}{2^{l}(l-k) ! k !} \exp [i x(l-2 k-A)]
$$

где

$$
A=\frac{m^{2}-\left(p_{0}+\mu\right)^{2}}{|e B|} .
$$


Если не выполняется условие

$$
R \equiv-2 k-A-1 \geqslant 0
$$

то контур интегрирования в (36) можно дополнить дугой бесконечного радиуса в нижней полуплоскости, интеграл по которой равен нулю. Так как в нижней полуплоскости подынтегральное выражение (36) не имеет особенностей, то члены с данными $k$ не дадут вклада в интеграл.

Если неравенство (37) выполняется, то контур интегрирования в (36) замыкается дугой бесконечного радиуса в верхней полуплоскости, и мы получаем вклад, определяемый суммой вычетов в точках $x=\pi(2 r-1) / 2$ :

$$
\begin{aligned}
& \sum_{r=-\infty}^{\infty} \oint_{C_{r}} \frac{d x}{\cos ^{l+1} x} e^{i x(B+l+1)}= \\
& \quad=\sum_{r=-\infty}^{\infty}(-1)^{r(l+1)} e^{i \pi(R+l+1)\left(r-\frac{1}{2}\right)} \oint_{C_{0}} \frac{d x}{\sin ^{l+1} x} e^{i x(R+l+1)} .
\end{aligned}
$$

Интегрирование по $x$ сводится к интегрированию по замкнутому контуру малого радиуса, охватываюшему точку $x=0$. Сумму по $r$ можно преобразовать к виду

$$
2 \sum_{r=-\infty}^{\infty} \delta(R-2 r)
$$

а из неравенства (37) следует, что индекс суммирования положителен, т.е. $r \geqslant 0$.

Учитывая значение интеграла

$$
\oint_{C_{0}} \frac{\sin ^{l+1} x}{e^{i x(2 r+l+1)}} d x=\pi(2 i)^{l+1} \frac{(r+l) !}{r ! l !},
$$

после простых преобразований находим

$$
J=-\frac{4 i \pi}{|e B|} \sum_{l=0}^{\infty} \sum_{k=0}^{l} \sum_{r=0}^{\infty} \frac{(-z)^{l}(-1)^{r+k}(r+l) !}{r ! l !(l-k) ! k !} \delta(R-2 r) .
$$

Так как в аргумент $\delta$-функции индексы $r$ и $k$ входят в виде суммы, введем новый индекс $n=r+k$ и изменим порядок суммирования в (39). Суммирование по $r, l$ приводит к соотношению

$$
\sum_{r=0}^{\infty} \sum_{l=0}^{\infty} \sum_{k=0}^{l} \frac{(-z)^{l}(-1)^{r+k}(r+l) !}{r ! l !(l-k) ! k !}=\sum_{n=0}^{\infty}(-1)^{n} I_{n n}(2 z)
$$

Здесь $I_{n k}(2 z)$ - функция Лагерра аргумента $2 z$,

$$
I_{n k}(2 z)=(n ! k !)^{-\frac{1}{2}} \exp (-z)(2 z)^{\frac{n-k}{2}} L_{k}^{n-k}(2 z),
$$


где $L_{s}^{k}(2 z)$ - обобшенный полином Лагерра, который в приведенном примере (так как $k=0)$ сводится к простому полиному Лагерра. Следовательно,

$$
J=-\frac{4 i \pi}{|e B|} \sum_{n=0}^{\infty}(-1)^{n} I_{n n}(2 z) \delta(R(k \rightarrow n)),
$$

при этом аргумент $\delta$-функции имеет вид

$$
R(k \rightarrow n)=-2 n-\frac{m^{2}-\left(p_{0}+\mu\right)^{2}}{|e B|}-1 .
$$

Так преобразуются члены в $\Delta S$ с $P^{0}$ и $M$, однако, как следует из (32), аргументы $\delta$-функций в этих выражениях будут отличаться от (43) на \pm 1 , а именно,

$$
R(k \rightarrow n)=-2 n-\frac{m^{2}-\left(p_{0}+\mu\right)^{2}}{|e B|}-1 \pm 1 .
$$

Аналогично преобразуется и член в $\Delta S$ с $\mathbf{P}$, только на последнем этапе вычислений при суммировании по $r, l$ используется формула

$$
\sum_{r=0}^{\infty} \sum_{l=0}^{\infty} \sum_{k=0}^{l} \frac{(-z)^{l}(-1)^{k}(r+l+1) !}{r !(l+1) !(l-k) ! k !}=\sum_{n=0}^{\infty} e^{-z} L_{n}^{1}(0) .
$$

Этот член будет содержать $\delta$-функцию аргумента (43). В результате получим следующее выражение для функции Грина уравнения Дирака при ненулевой плотности фермионов:

$$
\begin{aligned}
& S^{\mathrm{c}}\left(\bar{p}_{0}, \mathbf{p}, m\right) \equiv S_{\mathrm{v}}(p)+S_{\mathrm{p}}(p)=-\frac{i}{|e B|} \int_{0}^{\infty} \frac{d x}{\cos x}[(\gamma P)+M] \times \\
& \quad \times \exp \left[\frac{i x}{|e B|}\left(\left(\bar{p}_{0}+i \operatorname{sign}\left(\bar{p}_{0}\right) \epsilon\right)^{2}-m^{2}+i \delta_{e}-\mathbf{p}^{2} \frac{\operatorname{tg} x}{x}\right)\right]-2 i \pi[(\gamma \bar{P})+\bar{M}],
\end{aligned}
$$

где

$$
\begin{aligned}
\bar{P}^{0}= & \left(\bar{p}_{0}+m\right) \delta\left(\bar{p}_{0}^{2}-m^{2}\right) \exp \left(-\frac{\mathbf{p}^{2}}{|e B|}\right)+ \\
& +2 m \sum_{n=1}^{\infty}(-1)^{n} I_{n n}\left(\frac{\mathbf{p}^{2}}{|e B|}\right) \delta\left(\bar{p}_{0}^{2}-m^{2}-2 n|e B|\right), \\
\bar{M}= & \left(\bar{p}_{0}+m\right) \delta\left(\bar{p}_{0}^{2}-m^{2}\right) \exp \left(-\frac{\mathbf{p}^{2}}{|e B|}\right)+ \\
& +2 \bar{p}_{0} \sum_{n=1}^{\infty}(-1)^{n} I_{n n}\left(\frac{\mathbf{p}^{2}}{|e B|}\right) \delta\left(\bar{p}_{0}^{2}-m^{2}-2 n|e B|\right) .
\end{aligned}
$$

Члены, пропорциональные $\mathbf{P}$, содержат $\delta$-функцию аргумента (43). В рассматриваемой нами задаче при интегрировании с участием функции Грина их вклад равен нулю, поэтому мы их не приводим.

Представление (46) удобно для изучения вопроса о локализации особенностей функции Грина по переменной $p_{0}$. В частности, видно, что полюсы функции Грина по переменной $p_{0}$ лежат в точках $p^{0}= \pm E_{n}-\mu$, где $E_{n}$ - дискретные уровни Ландау

$$
p_{0}+\mu= \pm \sqrt{m^{2}+2 n|e B|}, \quad n=0,1, \ldots .
$$




\section{4. ТЕНЗОРНАЯ СТРУКТУРА ПОЛЯРИЗАЦИОННОГО ОПЕРАТОРА В КЭД $2+1$}

Поляризационный оператор (ПО) в постоянном и однородном внешнем поле диагонален по 3-импульсу фотона. В импульсном представлении он определяется формулой

$$
\Pi^{\mu \nu}(k)=i e^{2} \int \frac{d^{3} p}{(2 \pi)^{3}} \operatorname{tr}\left[\gamma^{\mu} S^{\mathrm{c}}(p+k, \mu, B) \gamma^{\nu} S^{\mathrm{c}}(p, \mu, B)\right] .
$$

Информацию о свойствах ПО, его тензорной структуре, а также о свойствах связанной с ПО так называемой полной функции Грина фотона $D^{\mu \nu}(p, q)$ можно получить исходя из ограничений, накладываемых требованиями релятивистской и калибровочной инвариантности теории, а также симметрией внешнего поля. Так, из релятивистской инвариантности следует, что ПО и функция Грина $D^{\mu \nu}(p, q)$ могут зависеть только от скаляров. Такие скаляры конструируются в результате различных сверток 3 -импульсов “фотона" $p, q$ и тензора внешнего поля. В постоянном и однородном магнитном поле ПО диагонален по 3-импульсу “фотона", поэтому $p=q$, и, следовательно, можно построить всего 3 независимых скаляра:

$$
p^{2}=p_{0}^{2}-\mathbf{p}^{2}, \quad s=p_{\mu} F^{\mu \nu} F_{\nu \rho} p^{\rho} \equiv B^{2}\left(p_{1}^{2}+p_{2}^{2}\right), \quad g=\frac{1}{2} F^{\mu \nu} F_{\mu \nu} \equiv B^{2} .
$$

Таким образом, в постоянном и однородном магнитном поле ПО (50) является функцией трех инвариантов (51).

Введем ортонормированную систему 3 -векторов $l_{i}^{\mu}[15]$,

$$
\begin{gathered}
l_{1}^{\mu}=\frac{F^{\mu \nu} p_{\nu}}{\sqrt{s}} \equiv \frac{1}{\sqrt{\mathbf{p}^{2}}}\left(0,-p_{2}, p_{1}\right), \\
l_{2}^{\mu}=e^{\mu \nu \rho} l_{0, \nu} l_{1, \rho} \equiv \frac{1}{\sqrt{p^{2} \mathbf{p}^{2}}}\left(\mathbf{p}^{2}, p_{0} p_{1}, p_{0} p_{2}\right) \\
l_{3}^{\mu}=\frac{p^{\mu}}{\sqrt{p^{2}}} \equiv \frac{1}{\sqrt{p^{2}}}\left(p_{0}, p_{1}, p_{2}\right),
\end{gathered}
$$

где $\mathbf{p}^{2}=p_{1}^{2}+p_{2}^{2}$, а $e^{\mu \nu \rho}-$ полностью антисимметричный единичный псевдотензор 3 -го ранга. Легко проверить, что

$$
g_{\mu \nu} l_{i}^{\mu} l_{k}^{\nu}=g_{i k}, \quad \sum_{i, k} g^{i k} l_{i}^{\mu} l_{k}^{\nu}=g^{\mu \nu},
$$

где $g^{\mu \nu}$ - тензор Минковского, а у тензора $g^{i j}$ отличные от нуля диагональные компоненты суть $g^{11}=g^{22}=-g^{33}=-1$. Вообше говоря, векторы $l_{i}^{\mu}, i=1,2$, не являются собственными векторами ПО, однако именно эти векторы образуют его тензорную структуру в однородном магнитном поле в КЭД $2+1$. Собственное значение ПО, соответствуюшее собственному вектору $l_{3}^{\mu}$, равно нулю, что является следствием калибровочной инвариантности,

$$
p_{\mu} \Pi^{\mu \nu}=\Pi^{\mu \nu} p_{\nu}=0 .
$$


В КЭД $2+1$ ПО (50) можно представить в виде разложения

$$
\Pi^{\mu \nu}=\left(g^{\mu \nu}-\frac{p^{\mu} p^{\nu}}{p^{2}}\right) \Pi_{0}+\mathbf{p}^{2} \Pi_{1} l_{1}^{\mu} l_{1}^{\nu}+i\left(l_{1}^{\mu} l_{2}^{\nu}-l_{2}^{\mu} l_{1}^{\nu}\right) C,
$$

где $\Pi_{0}, \Pi_{1}, C$ - скалярные функции, зависящие от инвариантов (51). Последний член в (55) есть так называемый индуцированный член Черна-Саймонса [19]. ПО можно представить в полностью поперечном виде

$$
\Pi^{\mu \nu}=\pi_{1} l_{1}^{\mu} l_{1}^{\nu}+\pi_{2} l_{2}^{\mu} l_{2}^{\nu}+i\left(l_{1}^{\mu} l_{2}^{\nu}-l_{2}^{\mu} l_{1}^{\nu}\right) C,
$$

если учесть, что

$$
-\left(g^{\mu \nu}-\frac{p^{\mu} p^{\nu}}{p^{2}}\right)=\sum_{i=1,2} l_{i}^{\mu} l_{i}^{\nu} .
$$

В выражении (56) $\pi_{1}, \pi_{2}$ - скалярные функции, определяемые функциями $\Pi_{0}, \Pi_{1}$. Следует подчеркнуть, что в отличие от случая КЭД ${ }_{3+1}$ ПО (56) в КЭД $2+1$ не симметричен по индексам $\mu, \nu$.

В КЭД $3+1$ собственные векторы ПО описывают поляризацию электромагнитных волн с импульсом $p^{\mu}$ во внешнем поле. Отметим, что если $p^{2}>0$, то векторы $l_{i}^{\mu}(i=1,2)$ пространственноподобны, т.е. $\left(l_{1}^{\mu}\right)^{2}<0,\left(l_{2}^{\mu}\right)^{2}<0$. Если же $p^{2}=0$, то вектор $l_{1}^{\mu}$ остается пространственноподобным, а $l_{2}^{\mu} \rightarrow p^{\mu} / \sqrt{p^{2}}$, так что "второй моды" в этом пределе не сушествует. Собственные векторы ПО в КЭД $2+1$ можно построить по аналогии со случаем $\mathrm{K} \beth_{3+1}$. Уравнения Максвелла для векторного потенциала электромагнитных волн (элементарных возбуждений) $A^{\mu}(p)$ с волновым вектором $p^{\mu}$, распространяющихся в некоторой эффективной среде (во внешнем поле), в линейном приближении

$$
p^{2} A^{\mu}(p)=-\int d^{3} q \Pi^{\mu \nu}(p, q, B) A_{\nu}(q)
$$

должны иметь решения вида

$$
A^{\mu}(p)=\sum_{k=1,2} e_{k}^{\mu}(p) \delta\left(p^{2}+\chi_{k}(p)\right)
$$

Мы учли свойство диагональности ПО в постоянном и однородном внешнем поле по 3-импульсу “фотона".

Подставляя (56) в (58), получаем уравнение для определения собственных векторов ПО

$$
\left[\pi_{1} l_{1}^{\mu} l_{1}^{\nu}+\pi_{2} l_{2}^{\mu} l_{2}^{\nu}+i\left(l_{1}^{\mu} l_{2}^{\nu}-l_{2}^{\mu} l_{1}^{\nu}\right) C\right] e_{\nu k}(p)=\chi_{k}(p) e_{k}^{\mu}(p) .
$$

Решение уравнения (60) ищем в виде

$$
e_{k}^{\mu}(p)=a_{k} l_{1}^{\mu}+i b_{k} l_{2}^{\mu},
$$

где $a_{k}(p), b_{k}(p)$ - амплитуды "собственных волн". В результате получим систему уравнений для определения коэффициентов $a_{k}, b_{k}$

$$
\begin{aligned}
\left(\chi_{k}+\pi_{1}\right) a_{k}-C b_{k} & =0, \\
-C a_{k}+\left(\chi_{k}+\pi_{2}\right) b_{k} & =0 .
\end{aligned}
$$


Введенные выше "правые" собственные векторы ПО нормированы следующим образом:

$$
e_{k}^{\mu *}(p) e_{j \mu}(p)=-\delta_{k j} .
$$

Векторы $e_{k}^{\mu *}(p)$ являются “левыми” собственными векторами ПО. Учитывая условие нормировки (63), находим

$$
a_{k}=\frac{1}{\sqrt{1+f^{2}}}, \quad b_{k}=\frac{f}{\sqrt{1+f^{2}}},
$$

где $f=C /\left(\chi_{k}+\pi_{2}\right)$. Собственные значения $\chi_{k}$ находятся из условия обрашения в нуль детерминанта системы уравнений (62):

$$
\left(\chi_{k}+\pi_{1}\right)\left(\chi_{k}+\pi_{2}\right)=C^{2}
$$

Если нас интересуют решения уравнений Максвелла, то, учитывая, что из (59) следует условие на собственные значения ПО в виде $\chi_{k}=p^{2}$, из (65) получим уравнение дисперсии "фотона"

$$
\left(p^{2}+\pi_{1}\right)\left(p^{2}+\pi_{2}\right)-C^{2}=0
$$

Дальнейший анализ свойств ПО в постоянном и однородном магнитном поле можно провести, вычислив функции $\pi_{1}, \pi_{2}, C$. Приведем только схему вычислений.

\section{5. ПОЛЯРИЗАЦИОННЫЙ ОПЕРАТОР В КЭД $2+1$ С НЕНУЛЕВОЙ ПЛОТНОСТЬЮ ФЕРМИОНОВ}

В однопетлевом приближении КЭД $2+1$ искомый ПО в постоянном и однородном магнитном поле удобно записать в виде двух слагаемых, описываюших вклады в ПО виртуальных (вклад вакуума) и реальных (вклад частиц) фермионов:

$$
\begin{aligned}
\Pi^{\mu \nu}(p)= & i e^{2} \int \frac{d^{3} q}{(2 \pi)^{3}} \operatorname{tr}\left[\gamma^{\mu} S^{\mathrm{c}}(q+p, \mu, B) \gamma^{\nu} S^{\mathrm{c}}(q, \mu, B)\right]= \\
= & i e^{2} \int \frac{d^{3} q}{(2 \pi)^{3}} \operatorname{tr}\left[\gamma^{\mu} S_{\mathrm{v}}(q+p, B) \gamma^{\nu} S_{\mathrm{v}}(q, B)\right]+ \\
& +i e^{2} \int \frac{d^{3} q}{(2 \pi)^{3}} \operatorname{tr}\left[\gamma^{\mu} S_{\mathrm{v}}(q+p, \mu, B) \gamma^{\nu} S_{\mathrm{p}}(q, \mu, B)+\right. \\
& \left.+\gamma^{\mu} S_{\mathrm{p}}(q+p, \mu, B) \gamma^{\nu} S_{\mathrm{v}}(q, \mu, B)\right]
\end{aligned}
$$

Первое слагаемое правой части последнего равенства формулы (67) дает вклад вакуума в ПО, члены второго слагаемого дают вклады, связанные с присутствием реальных фермионов.

Рассмотрим вначале вакуумную часть ПО. Учтем следующие легко проверяемые соотношения:

$$
\begin{gathered}
\operatorname{tr}\left[\gamma^{\mu} \gamma^{\nu}\right]=2 g^{\mu \nu}, \quad \operatorname{tr}\left[\gamma^{\mu} \gamma^{\nu} \gamma^{\rho}\right]=-2 i e^{\mu \nu \rho}, \operatorname{tr}\left[\gamma^{\mu} \gamma^{\rho} \gamma^{\epsilon} \gamma^{\nu}\right]=2 e^{\mu \rho \sigma} e_{\sigma}^{\epsilon \nu} \\
e^{\mu \rho \sigma} e_{\sigma}^{\epsilon \nu}=g^{\mu \epsilon} g^{\rho \nu}-g^{\mu \nu} g^{\rho \epsilon}
\end{gathered}
$$


Вместо переменных $x_{1}$ и $x_{2}$, от которых зависят вакуумные части функций Грина (34), удобно ввести переменные

$$
x=x_{1}+x_{2}, \quad v=\frac{x_{1}-x_{2}}{x_{1}+x_{2}}=\frac{x_{1}-x_{2}}{x},
$$

области изменения которых суть $0 \leqslant x<\infty$ и $-1 \leqslant v \leqslant 1$.

В результате вычислений мы представили вакуумную часть ПО в следуюшем (полностью поперечном) виде:

$$
\begin{gathered}
\Pi_{\mathrm{v}}^{\mu \nu}(p, B)=\frac{i^{\frac{1}{2}} e^{2}}{16 \pi^{\frac{3}{2}} \sqrt{|e B|}} \int_{0}^{\infty} \frac{\sqrt{x} d x}{\sin x} \int_{-1}^{1} d v\left[\pi_{1} l_{1}^{\mu} l_{1}^{\nu}+\pi_{2} l_{2}^{\mu} l_{2}^{\nu}-i e^{\mu \nu \rho} p_{\rho} C^{\prime}\right] \times \\
\quad \times \exp \left[\frac{i x}{|e B|}\left(\frac{p_{0}^{2}\left(1-v^{2}\right)}{4}-\frac{\mathbf{p}^{2} \sin \left(\frac{x}{2}(1+v)\right) \sin \left(\frac{x}{2}(1-v)\right)}{x \sin x}-m^{2}+i \epsilon\right)\right],
\end{gathered}
$$

где

$$
\begin{aligned}
\pi_{1}= & p_{0}^{2}(\cos (v x)-v \operatorname{ctg} x \sin (v x))- \\
& -\mathbf{p}^{2} \frac{1}{\sin ^{2} x}[v \sin (2 v x) \sin x-2 \cos (v x)+\cos x(1+\cos (2 v x))], \\
\pi_{2}= & p^{2}(\cos (v x)-v \operatorname{ctg} x \sin (v x)), \\
C^{\prime}= & 2 m \cos (v x) .
\end{aligned}
$$

При получении формулы (71) в новом виде мы использовали соотношение

$$
l_{1}^{\mu} l_{2}^{\nu}-l_{2}^{\mu} l_{1}^{\nu}=-\frac{e^{\mu \nu \rho} p_{\rho}}{\sqrt{p^{2}}} .
$$

Из формул (71)-(74) видно, что вакуумную часть ПО в постоянном и однородном магнитном поле можно рассматривать как функцию $p_{0}^{2}, \mathbf{p}^{2}$ и безразмерного параметра $\kappa=e \hbar B / m^{2} c^{3}$, в который входят заряд фермиона, внешнее поле и постоянная Планка. Из (71) видно также, что ПО неаналитичен по параметру $\kappa$. Отметим, что неаналитичность ПО по заряду, внешнему полю и постоянной Планка проявляется одновременно. Неаналитичность в выражениях для вероятностей различных квантовых процессов по заряду в присутствии внешних постоянных электромагнитных полей была исследована в ряде статей Ритуса (см. [18] и цитируемую там литературу).

Из (71) нетрудно получить выражение для ПО в КЭД $2+1$ в отсутствие внешнего магнитного поля, т.е. в пределе $e B \rightarrow 0$. В этом пределе основной вклад в интеграл по переменной $x$ дает область малых значений $x$, так что все тригонометрические функции подынтегрального выражения ПО можно разложить в ряды, сохранив только основные члены. В итоге мы получим

$$
\begin{aligned}
\Pi^{\mu \nu}(p)= & \frac{i^{\frac{1}{2}} e^{2}}{16 \pi^{\frac{3}{2}}} \int_{0}^{\infty} \frac{d s}{\sqrt{s}} \int_{-1}^{1} d v\left[\left(1-v^{2}\right)\left(g^{\mu \nu} p^{2}-p^{\mu} p^{\nu}\right)-2 i m e^{\mu \nu \rho} p_{\rho}\right] \times \\
& \times \exp \left[i s\left(\frac{p^{2}\left(1-v^{2}\right)}{4}-m^{2}+i \epsilon\right)\right] .
\end{aligned}
$$

6 Теоретическая и математическая физика, т. 125, № 1, 2000 г. 
ПО в отсутствие внешних полей является функцией только одного скаляра $p^{2}$. Обозначив переменную $p^{2} / m^{2}$ через $t$, можно аналогично случаю КЭД $3+1$ изучить аналитические свойства ПО в плоскости комплексного $t$. В частности, из (76) видно, что на отрицательной вешественной полуоси ПО является вешественной функцией, а особые точки этой функции лежат на положительной вешественной полуоси переменной $t$ при значениях, являющихся пороговыми для рождения виртуальным “фотоном” различных совокупностей реальных частищ. Точка $t=4$ является пороговой для рождения пары фермионов (частицы и античастицы); области $t<0$ и $t>4$ являются физическими областями, а область $0 \leqslant t \leqslant 4-$ нефизической.

Интегрирование в формуле (76) легко выполнить, считая $t<0$ (тогда мнимую добавку в показателе экспоненты подынтегрального выражения можно опустить). Окончательное выражение для ПО в отсутствие магнитного поля можно представить в виде

$$
\begin{aligned}
\Pi^{\mu \nu}(p)= & \frac{e^{2}}{8 \pi|m|}\left\{\left(g^{\mu \nu} p^{2}-p^{\mu} p^{\nu}\right)\left[\frac{2}{\sqrt{-t}}\left(\frac{1}{2}+\frac{2}{t}\right) \operatorname{arctg} \frac{\sqrt{-t}}{2}+\frac{1}{\sqrt{-t}}\right]-\right. \\
& \left.-i m e^{\mu \nu \rho} p_{\rho} \frac{4}{\sqrt{-t}} \operatorname{arctg} \frac{\sqrt{-t}}{2}\right\},
\end{aligned}
$$

где $t=p^{2} / m^{2}$. Для получения выражения ПО, справедливого во всей области значений переменной $t$, формулу (77) можно аналитически продолжить. При этом удобно ввести новую переменную $z$, определив ее [28] как $t=-(1-z)^{2} / z$.

Рассмотрим теперь члены во втором слагаемом правой части последнего равенства формулы (67). Эти члены описывают вклад реальных фермионов в ПО. Во втором члене этого слагаемого заменим $q$ на $q-p$ и преобразуем его к виду

$$
i e^{2} \int \frac{d^{3} q}{(2 \pi)^{3}} \operatorname{tr}\left[\gamma^{\mu} S_{\mathrm{v}}(q+p, \mu, B) \gamma^{\nu} S_{\mathrm{p}}(q, \mu, B)+\gamma^{\mu} S_{\mathrm{p}}(q, \mu, B) \gamma^{\nu} S_{\mathrm{v}}(q-p, \mu, B)\right] .
$$

На примере типичных интегралов в формуле (78) поясним, как вьполнить интегрирование по q.

Имеем интеграл

$$
\int \frac{d^{3} q}{(2 \pi)^{3}} \exp \left[\frac{i x}{b}\left(\left(\bar{q}_{0} \pm p_{0}\right)^{2}-m^{2}-(\mathbf{q} \pm \mathbf{p})^{2} \frac{\operatorname{tg} x}{x}\right)\right] I_{n n}\left(\frac{\mathbf{q}^{2}}{b}\right) \delta\left(\bar{q}_{0}^{2}-m^{2}-2 n b\right),
$$

где мы обозначили $b=|e B|$, а знаки \pm в показателе экспоненты появляются при интегрировании первого и второго членов формулы (78), соответственно. Преобразуем интегралы, содержащие двумерные векторы $\mathbf{p}, \mathbf{q}$,

$$
\begin{aligned}
\int d \mathbf{q} \exp \left[-i(\mathbf{q} \pm \mathbf{p})^{2} b^{-1} \operatorname{tg} x\right] I_{n n}\left(\frac{\mathbf{q}^{2}}{b}\right)= \\
=\int d \mathbf{q} \exp \left[-i\left(\mathbf{q}^{2} \pm 2 \mathbf{q} \mathbf{p}+\mathbf{p}^{2}\right) b^{-1} \operatorname{tg} x\right] I_{n n}\left(\frac{\mathbf{q}^{2}}{b}\right) .
\end{aligned}
$$

Вычисление проводим в полярных координатах. Учтем соотношение [29]

$$
\exp \left[\mp\left(i 2 q p b^{-1} \operatorname{tg} x\right) \cos \vartheta\right]=\sum_{l=-\infty}^{\infty}(\mp i)^{l} J_{l}(q y) \exp [\mp(i \vartheta)]
$$


где $\vartheta$ - угол между векторами $\mathbf{p}$ и $\mathbf{q}, J_{l}(z)$ - функция Бесселя целого индекса $l$ и мы обозначили $y=2 p \operatorname{tg} x / b$. Направив полярную ось вектора q по вектору $\mathbf{p}$, в результате интегрирования по полярному углу вектора q получим $2 \pi \delta_{l 0}$. Суммируя по $l$, найдем $J_{0}(q y)$. Интегрирование по модулю вектора q выполним с помощью формулы [29]

$$
\int_{0}^{\infty} d z z e^{-\frac{a z^{2}}{2}} J_{0}(z y) L_{n}\left(\frac{c z^{2}}{2}\right)=\frac{(a-c)^{n}}{a^{n+1}} e^{-\frac{y^{2}}{2 a}} L_{n}\left(\frac{c y^{2}}{2 a(c-a)}\right) .
$$

Вводя переменную $z=q / \sqrt{b}$ и учитывая, что в нашем случае $a=2(1+i \operatorname{tg} x)=$ $2 e^{i x} / \cos x, c-a=2 e^{-i x} / \cos x$, получим

$$
\begin{aligned}
\int d \mathbf{q} \exp [ & \left.-i\left(\mathbf{q}^{2} \pm 2 \mathbf{q p}+\mathbf{p}^{2}\right) b^{-1} \operatorname{tg} x\right] I_{n n}\left(\frac{\mathbf{q}^{2}}{b}\right)= \\
= & \pi b(-1)^{n} \exp \left\{-2 i x\left(n+\frac{1}{2}\right)\right\} \times \\
& \times \exp \left\{-\mathbf{p}^{2} \frac{(1-\cos 2 x+i \sin 2 x)}{2 b}\right\} \cos x L_{n}\left(\frac{2 \mathbf{p}^{2} \sin ^{2} x}{b}\right) .
\end{aligned}
$$

Интегрирование по $q_{0}$ проводится с помошью $\delta$-функций. Заметим, что согласно замечанию, сделанному в разделе 3 (после формулы (48)), вклад дают только члены, содержашие $\bar{P}_{0}$ и $\bar{M}$, которые определены формулами (47) и (48). После интегрирования по q типичные интегралы по $q_{0}$ принимают вид

$$
\begin{aligned}
\left(\frac{\bar{P}^{0}}{\bar{M}}\right)= & =\cos x \exp \left\{-\frac{\mathbf{p}^{2}(1-\cos 2 x+i \sin 2 x)}{2 b}\right\} \int d q_{0} \exp \left\{\frac{i x\left[\left(p_{0} \pm \bar{q}_{0}\right)^{2}-m^{2}+i \epsilon\right]}{b}\right\} \times \\
& \times\left[\left(\begin{array}{c}
1 \\
1
\end{array}\right)\left(\bar{q}_{0}+m\right) \delta\left(\frac{\bar{q}_{0}^{2}-m^{2}}{b}\right) e^{-i x}+\right. \\
& \left.+2\left(\begin{array}{c}
m \\
\bar{q}_{0}
\end{array}\right) \sum_{n=1}^{\infty} e^{-2 i x\left(n+\frac{1}{2}\right)} L_{n}\left(\frac{2 \mathbf{p}^{2} \sin ^{2} x}{b}\right) \delta\left(\frac{\bar{q}_{0}^{2}-m^{2}-2 n|e B|}{b}\right)\right]
\end{aligned}
$$

Здесь верхний знак в показателе экспоненты относится к первому члену формулы (78), а нижний - ко второму.

После интегрирования по $q_{0}$ экспоненты подынтегрального выражения принимают ВИД

$$
\exp \left\{\frac{i x\left(p_{0}^{2} \pm 2 p_{0} E_{n}-b\right)}{b}-\frac{\mathbf{p}^{2}(1-\cos 2 x+i \sin 2 x)}{2 b}-\epsilon x\right\},
$$

где $E_{n}=\sqrt{m^{2}+2|e B| n}$ - релятивистские уровни энергии частиц в однородном магнитном поле (уровни Ландау). Мы предполагаем, что $\mu>m$. Это соответствует случаю, когда уровни Ландау заполнены частицами, а реальные античастицы отсутствуют. Следовательно, дельта-функции сушественны только в том случае, когда $\mu>m$, что дает ограничение на индекс суммирования и фактически оставляет только один член ряда по $n$. Предполагается также, что $\mu \neq m, E_{n}$. Это означает, что среди $n$ уровней Ландау нет уровней, заполненных частично. Все $n$ уровней Ландау заполнены полностью. 
В результате вычислений выражение для части ПО, вклад в которую дают реальные частицы, можно получить в виде

$$
\begin{aligned}
\Pi_{\mathrm{p}}^{\mu \nu}(p, B)= & \frac{e^{2}}{2 \pi} \int_{0}^{\infty} d x\left[\pi_{p} p^{2} l_{2}^{\mu} l_{2}^{\nu}+i e^{\mu \nu \rho} p_{\rho} m C_{p}\right] \times \\
& \times \exp \left\{\frac{i x p_{0}^{2}}{b}-i x-\frac{\mathbf{p}^{2}(1-\cos 2 x+i \sin 2 x)}{2 b}-\epsilon x\right\},
\end{aligned}
$$

где

$$
\begin{aligned}
\pi_{p} & =\sin \left(\frac{2 m x p_{0}}{b}\right)+\frac{m}{E_{n}} L_{n}\left(2 b^{-1} \mathbf{p}^{2} \sin ^{2} x\right) \sin \left(2 E_{n} x p_{0} b^{-1}\right)\left[\frac{\mu^{2}-m^{2}}{2|e B|}\right], \\
C_{p} & =i \cos \left(\frac{2 m x p_{0}}{b}\right)+i L_{n}\left(2 b^{-1} \mathbf{p}^{2} \sin ^{2} x\right) \cos \left(2 E_{n} x p_{0} b^{-1}\right)\left[\frac{\mu^{2}-m^{2}}{2|e B|}\right] .
\end{aligned}
$$

Первые члены в формулах (87), (88) описывают вклад основного уровня Ландау $($ с $n=0), \quad[z]$ обозначает целую часть $z$. Аргумент $\left(\mu^{2}-m^{2}\right) / 2|e B|$ функции $\left[\left(\mu^{2}-m^{2}\right) / 2|e B|\right]$ не должен быть равен целому числу, так как при этих значениях аргумента функция становится неоднозначной.

Полный ПО в постоянном и однородном магнитном поле в однопетлевом приближении $\mathrm{K} Э Д_{2+1}$ можно записать в виде суммы двух слагаемых, представляющих вклады вакуума (формула (71)) и реальных частищ (формула (86)):

$$
\Pi_{\mathrm{t}}^{\mu \nu}(p, B)=\Pi_{\mathrm{v}}^{\mu \nu}(p, B)+\Pi_{\mathrm{p}}^{\mu \nu}(p, B) .
$$

\section{6. ОБСУЖДЕНИЕ РЕЗУЛЬТАТОВ}

Так как функции $\pi_{1}, \pi_{2}$ и $C^{\prime}$ в формулах (72)-(74) являются функциями $p_{0}, \mathbf{p}^{2}, B$ и $\mu$ и оператор $\Pi_{\mu \nu}$ конечен в длинноволновом пределе $p \rightarrow 0$, то единственный член, который не исчезает в этом пределе в выражении для ПО, - это антисимметричный член. Соответствующий член в эффективном лагранжиане - это индуцированный член Черна-Саймонса $[19,30]$. Действительно, в матрище квантовой электродинамики член второго порядка содержит слагаемые, отвечающие только собственным энергиям фермиона и фотона, причем поправка к лагранжиану электромагнитного поля в однопетлевом приближении КЭД определяется членом [31]

$$
S_{2}=-i A_{\mu}(x) \Pi^{\mu \nu}(x, y) A_{\nu}(y) .
$$

Коэффициент при члене $i e^{\mu \nu \rho} p_{\rho}$ при $p_{\mu}=0$ в выражении для ПО называют индуцированным коэффициентом Черна-Саймонса. Известно [32], что ненулевой вклад в коэффициент Черна-Саймонса в пределе $p \rightarrow 0$ возникает только в однопетлевом приближении $\mathrm{K} Э Д_{2+1}$.

В рассматриваемой модели КЭД $2+1$ вклад вакуума в этот коэффициент в отсутствие магнитного поля есть

$$
\gamma=-(\operatorname{sign} m) \frac{e^{2}}{4 \pi} .
$$


В присутствии внешнего постоянного магнитного поля индуцированный коэффициент Черна-Саймонса также вычисляется точно,

$$
\gamma=\frac{i^{\frac{1}{2}} e^{2} m}{8 \pi^{\frac{3}{2}} \sqrt{|e B|}} \int_{0}^{\infty} \frac{\sqrt{x} d x}{\sin x} \int_{-1}^{1} \cos v x \exp \left(-\frac{i x m^{2}}{|e B|}\right) d v=-(\operatorname{sign} m) \frac{e^{2}}{4 \pi}
$$

Заметим, что в последнем равенстве формулы (92) вместо знаковой функции sign $m$ можно поставить функцию $\operatorname{sign}(e B)$, так как во внешнем электромагнитном поле античастищы можно отличить от частиц и по знаку заряда. Вклад в индуцированньй коэффициент Черна-Саймонса в этом случае дают только вакуумные фермионы низшего уровня Ландау, поскольку вклады состояний с $n \geqslant 1$ должны обращаться в нуль в пределе $p_{\mu}=0$. Возникновение ненулевого индуцированного коэффициента Черна-Саймонса в отсутствие реальных частиц - это проявление эффекта поляризации вакуума внешним электромагнитным полем.

При ненулевой плотности частиц полньй вклад в индуцированный коэффициент Черна-Саймонса определяется суммой вакуумного члена и коэффициента при члене $i e^{\mu \nu \rho} p_{\rho}$ для $p_{\mu}=0$ в формуле (86). Этот коэффициент можно вычислить точно, так что в результате (полный) индуцированный коэффициент Черна-Саймонса можно представить в следуюшем виде:

$$
\begin{aligned}
\gamma(\mu, B)= & -(\operatorname{sign} m) \frac{e^{2}}{4 \pi} \theta(|m|-\mu)+ \\
& +\operatorname{sign}(e B) \frac{e^{2}}{2 \pi}\left(\frac{1}{2}+\left[\frac{\mu^{2}-m^{2}}{2|e B|}\right] \theta(\mu-|m|)\right) .
\end{aligned}
$$

Здесь $\theta(x)$ - функция Хэвисайда, [·] обозначает целую часть числа и предполагается, что аргумент $\left(\mu^{2}-m^{2}\right) / 2|e B|$ функции $\left[\left(\mu^{2}-m^{2}\right) / 2|e B|\right]$ не равен целому числу. Отметим, что полный коэффициент Черна-Саймонса меняет знак по отношению к вакуумному коэффициенту, если заполнен хотя бы один уровень Ландау.

Появление в эффективном лагранжиане члена Черна-Саймонса означает, что фотон приобретает "массу" динамически. Это можно увидеть из общего анализа уравнений дисперсии для квантованного поля излучения, проведенного выше с помощью ПО. Полезно, однако, связать это явление непосредственно с эффективным лагранжианом. Первая пара уравнений Максвелла не меняется, так как не меняется связь напряженностей полей с потенциалом $A_{\mu}$. Вторая же пара уравнений получается при варьировании по $A_{\mu}$ действия

$$
S=-\int d^{3} x\left\{\frac{1}{4} F_{\mu \nu} F^{\mu \nu}+\frac{1}{2} \gamma(\mu, B) e^{\mu \nu \rho} F_{\mu \nu} A_{\rho}\right\}
$$

что приводит к уравнению

$$
\partial_{\nu} F^{\nu \mu}-\gamma(\mu, B) e^{\mu \nu \rho} \partial_{\nu} A_{\rho}=0
$$

для компонент электромагнитного поля $F^{\mu \nu}$. 
В рассмотренной модели при определенных условиях может иметь место релятивистский целочисленный квантовый эффект Холла. Индуцированный коэффищиент Черна-Саймонса в этом случае имеет физический смысл недиагональной электрической проводимости [17]. Определенная таким образом электрическая проводимость является кусочно-непрерывной функцией магнитного поля при постоянном химическом потенциале (и наоборот), терпяшей разрывы при $\mu=-m, \pm E_{n}$. Поэтому для физических приложений интерес представляет обобшение полученного выражения для коэффициента Черна-Саймонса на случай конечной (ненулевой) температуры $T$.

Для вычисления коэффициента Черна-Саймонса при $T \neq 0$ нам нужна функция Грина идеального релятивистского ферми-газа в постоянном магнитном поле при конечной температуре. Как показано в работе [25], функция Грина свободного идеального нерелятивистского ферми-газа при конечной температуре совпадает с соответствуюшей функцией Грина (в импульсном представлении) при нулевой температуре с точностью до множителя $\operatorname{th}\left(p_{0} / 2 T\right)$, который следует поставить перед $\delta\left(p_{0}-\mu-\mathbf{p}^{2} / 2 m\right)$. Можно показать, что функция Грина идеального релятивистского ферми-газа в постоянном магнитном поле при $T \neq 0$ определяется формулой (46), в которой перед членом, пропорциональным $\delta$-функции, следует ввести аналогичный множитель $\operatorname{th}\left(p_{0} / 2 T\right)$. В результате такой замены для коэффищиента Черна-Саймонса при $T \neq 0$ получим

$$
\begin{aligned}
\gamma(\mu, B, T)= & -(\operatorname{sign} m) \frac{e^{2}}{4 \pi} \theta(|m|-\mu)+ \\
& +\operatorname{sign}(e B) \frac{e^{2}}{4 \pi} \sum_{n=0}^{\infty}\left[\operatorname{th}\left(\frac{\mu-E_{n}}{2 T}\right)+\operatorname{th}\left(\frac{\mu+E_{n}}{2 T}\right)\right] .
\end{aligned}
$$

Здесь первый (не зависящий от температуры) член дает вклад вакуума. Отметим, что при $T=0$ полученная формула переходит в формулу (93).

Благодарности. В заключение я хотел бы поблагодарить Чон Лин Xo (Choon-Lin Но) за полезные дискуссии и ценные замечания.

\section{Список литературы}

[1] A. M. J. Schakel, G. W. Semenoff. Phys. Rev. Lett. 1991. V. 66. P. 2653.

[2] The Quantum Hall Effect. 2nd ed. Eds. R. E. Prange, S.M. Girvin. New York: Springer-Verlag, 1990.

[3] F. Wilczek. Fractional Statistics and Anyon Superconductivity. Singapore: World Scientific, 1990.

[4] A. M. J. Schakel. Phys. Rev. D. 1991. V. 43. P. 1428.

[5] A. Neagu, A. M. J. Schakel. Phys. Rev. D. 1993. V. 48. P. 1785.

[6] J. M. Leinaas, J. Myrheim. Nuovo Cim. B. 1977. V. 37. P. 1.

[7] G. A. Goldin, R. Menikoff, D. H. Sharp. J. Math. Phys. 1981. V. 22. P. 1664.

[8] F. Wilczek. Phys. Rev. Lett. 1982. V. 48. P. 1144; V. 49. P. 957.

[9] Y. Hosotani. Phys. Lett. B. 1993. V. 319. P. 332; Phys. Rev. D. 1995. V. 51. P. 2022.

[10] V.R. Khalilov, C. L. Ho. Mod. Phys. Lett. A. 1998. V. 13. P. 615.

[11] B.P. Халилов. ТМФ. 1999. Т. 119. C. 105; C.L. Ho, V.R. Khalilov. Phys. Rev. A. 2000. V. 61. P. 032104.

[12] A. V. Turbiner. Commun. Math. Phys. 1988. V. 118. P. 467; M. A. Shifman, A. V. Turbiner. Commun. Math. Phys. 1989. V. 126. P. 347. 
ПОЛЯРИЗАЦИЯ ВАКУУМА $(2+1)$-МЕРНОЙ КВАНТОВОЙ ЭЛЕКТРОДИНАМИКИ 151

[13] V.R. Khalilov, C. L. Ho. Phys. Rev. D. 1999. V. 60. P. 033003.

[14] В. И. Рumyс. ЖЭТФ. 1969. Т. 57. С. 2176.

[15] И. А. Баталин, А.Е. Шабад. ЖЭТФ. 1971. Т. 60. С. 894.

[16] А. А. Гриб, С. Г. Мамаев, В. М. Мостепаненко. Вакуумные квантовые эффекты в сильных полях. М.: Энергоатомиздат, 1988.

[17] V. Zeitlin. Phys. Lett. B. 1995. V. 352. P. 422.

[18] В. И. Рumyc. Тр. ФИАН СССР. 1979. Т. 111. С. 5.

[19] S. Deser, R. Jackiw, S. Templeton. Phys. Rev. Lett. 1982. V. 48. P. 975; Ann. Phys. (N. Y. ). 1982. V. 140. P. 372.

[20] И. М. Тернов, В. Ч. Жуковский, А. В. Борисов. Квантовые процессы в сильном внешнем поле. М.: МГУ, 1989.

[21] Г. Бейтмен, А. Эрдейи. Высшие трансцендентные функции. Т. 2. М.: Наука, 1974.

[22] V. A. Fock. Sow. Phys. 1937. V. 12. P. 404.

[23] J. Schwinger. Phys. Rev. 1951. V. 82. P. 664.

[24] В. Р. Халилов. ТМФ. 1999. Т. 121. С. 412.

[25] Е. М. Лифиич, Л. П. Питаевский. Статистическая физика. Ч. 2. М.: Наука, 1978.

[26] A. E. Шабад. Особенности поляризационного оператора фотона в магнитном поле. Препринт ФИАН № 60. М.: ФИАН, 1974.

[27] А.Е. Лобанов, В.Р. Халилов. ЖЭТФ. 1979. Т. 77. С. 548.

[28] В. Б. Берестецкий, Е. М. Лифиии, Л. П. Питаевский. Квантовая электродинамика. М.: Наука, 1980.

[29] И. С. Градштейн, И. М. Рыжик. Таблицы интегралов, сумм, рядов и произведений. М.: ГИФМЛ, 1963.

[30] A. Redlich. Phys. Rev. D. 1984. V. 29. P. 2366

[31] Н. Н. Боголюбов, Д. В. Ширков. Квантовые поля. М.: Наука, 1980.

[32] T. Bernstein, A. Lee. Phys. Rev. D. 1985. V. 32. P. 1020; S. Coleman, B. Hill. Phys. Lett. B. 1985. V. 159. P. 184; Y. Nagahami. Z. Phys. C. 1986. V. 37. P. 583.

Поступила в редакцию 21.II.2000 г. 